\title{
Technology Based Teaching (TBT): A Transformational study with Database Management System
}

\author{
Nishant Doshi \\ Department of Computer Science and Engineering, Pandit Deendayal Petroleum University \\ doshinikki2004@gmail.com
}

\begin{abstract}
In this 21 st century, edifying is not inhibited to pen and board method as it used to be an efficacious (or verbalize only) source of communication in the earlier days. Nowadays, its span from four walls of class to any corner of the world. Students can optically discern, learn, etc. from anywhere of the world's corner. In this paper we have given our experiments with Undergrad subject Database Management Systems (DBMS) predicated on the technology implements like crossword, scramble word, findword, Kahoot and so on. In the cessation, we have done the survey in the respective class to get the feedback of these technology.
\end{abstract}

Keywords: Teaching, Transformation, DBMS, Kahoot, crossword, Findword

\section{Introduction}

From antediluvian time, several things emerged to as it afore relishes mobile, communication, attire, etc. On the other side, several things not yet transmuted i.e. edifying in school, higher educations, etc. In edifying, the edifier utilizes the sundry medium of communication like chalk, pen, board, etc. to edify the

\footnotetext{
Nishant Doshi

Department of Computer Science and Engineering,

Pandit Deendayal Petroleum University

doshinikki2004@gmail.com
}

students. However, with the recent development of the digital technology revolution, students cannot be constrained to the older method of edifying. On the other hand, every student's ingenuity required to be nurtured to make the innovation in the subject. As well verbalized that there is a different path which leads to the same goal. Same, in edifying there is a desideratum for sundry other technological and innovation predicated method to avail the understood of topics and clear the fundamentals.

In our research, we do the analysis of student demeanors and ascertained that the other activities than edifying supposed to be cultivated in developing the edification method. Like, students are quite magnetized to crossword, anagram, Sudoku, etc. which comes in a newspaper on a circadian substructure. Thus, we concluded that the hobbies of students can be acclimated to get them understood the concept of the subject.

In our literature survey, we study several technologies like Moodle, Google classroom, etc. We additionally studies several papers cognate to them i.e. (Borich, 1998), (Dianat, 2019), (Tutu, 2019), (Rao,2013),

(Welch et al. 2001), (Prince and Felder, 2006), (Wankat et al, 2002), (Kapranos and Tsakiropoulos, 2008), (Houghton, 2004), (Goodhew, 2010) and (Atasi and Deepsikha, 2016). However, we find the fundamental research gap in this literature is to not cultivate the hobbies of a student as a medium for 
edifying. Thus, we mentally conceived of converting the most used habits of students in the edification and prosperously integrate into the undergrad course Database Management Systems (DBMS). After each technology we have taken the feedback from students and to our surprise, they quite relish this to brush up their fundamentals in the subject.

\section{A. Our Contribution}

As this paper is the outcome of our experiments with students from the last two years, we have done the following technology contribution towards teaching methods

- We have to use the crossword technique

- We have used the findword technique

- We have used scramble word technique

- We have used Kahoot as a digital technique

\section{B. Paper Organization}

The entire document is organized as follows. In starting sections (i.e. section $2-4$ ) we have focused on the non- digital technique while in a later section (i.e. section 5). Finally, we have given the conclusions and references are at the end. For section $2-4$ we have utilized the help of Teacher's corner (https://worksheets.theteacherscorner.net/makeyour-own/crossword/). Finally, in Table 1, we have given the summery of our feedback on this technology.

\section{Crossword}

Crossword is a technique in which learner will be given horizontal and vertical lines and that supposed to be matched in accordance. In Fig 1, we have given the sample blank crossword and in Fig 2. We have the crossword with answers.

\section{Findword}

In the findword, learners will be given words in rows and column and they have to find the correct sequence in a row, column or diagonal including a reverse in all of them. Fig.3 shows the example of findword and Fig. 4 shows the solution to that findword.

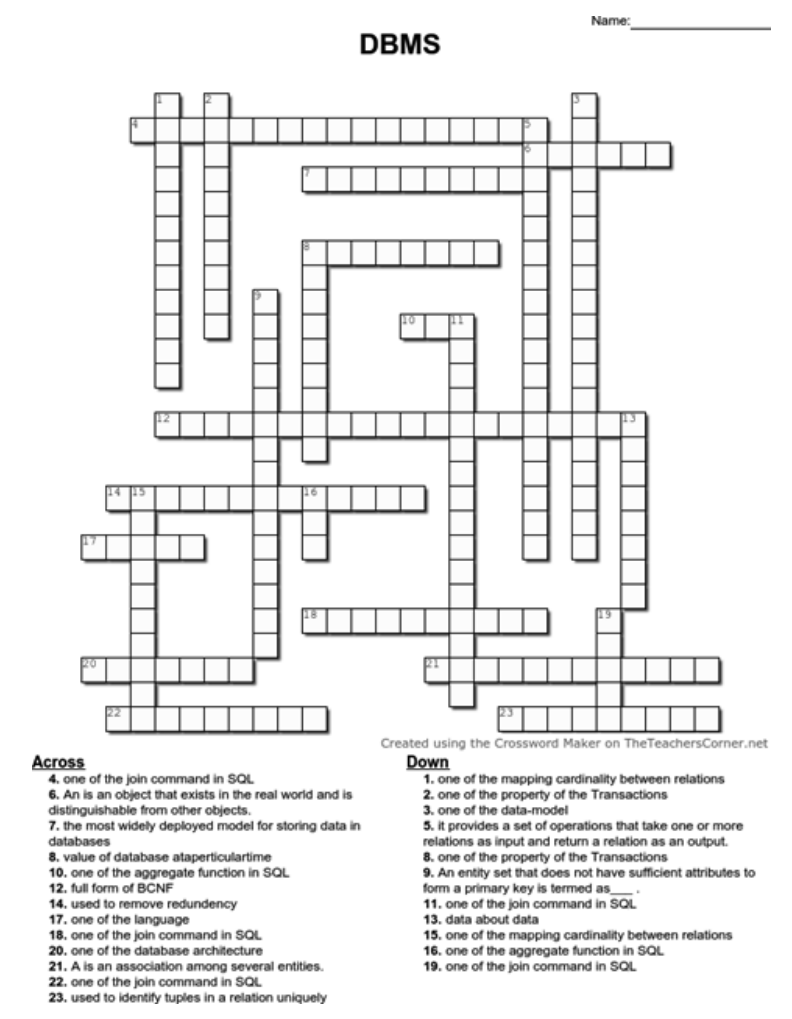

Fig. 1 : Crossword

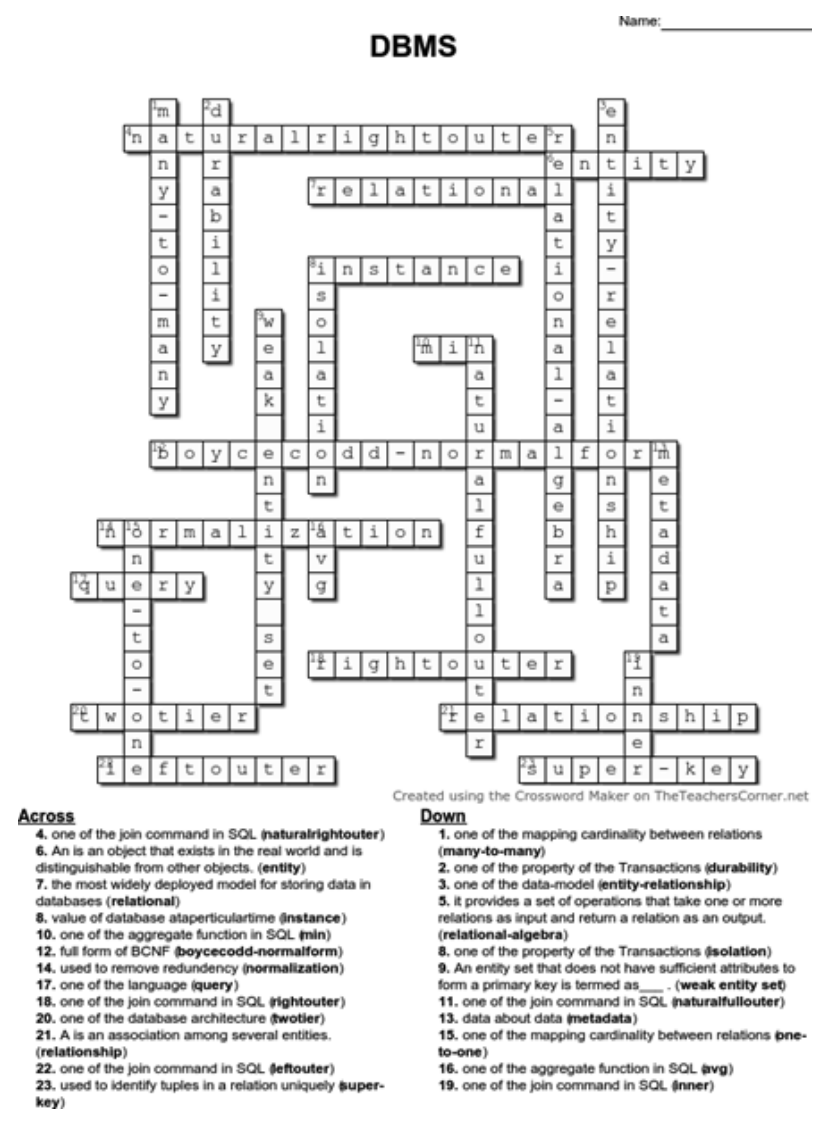

Fig 2 : Crossword with Answers 


\section{DBMS}

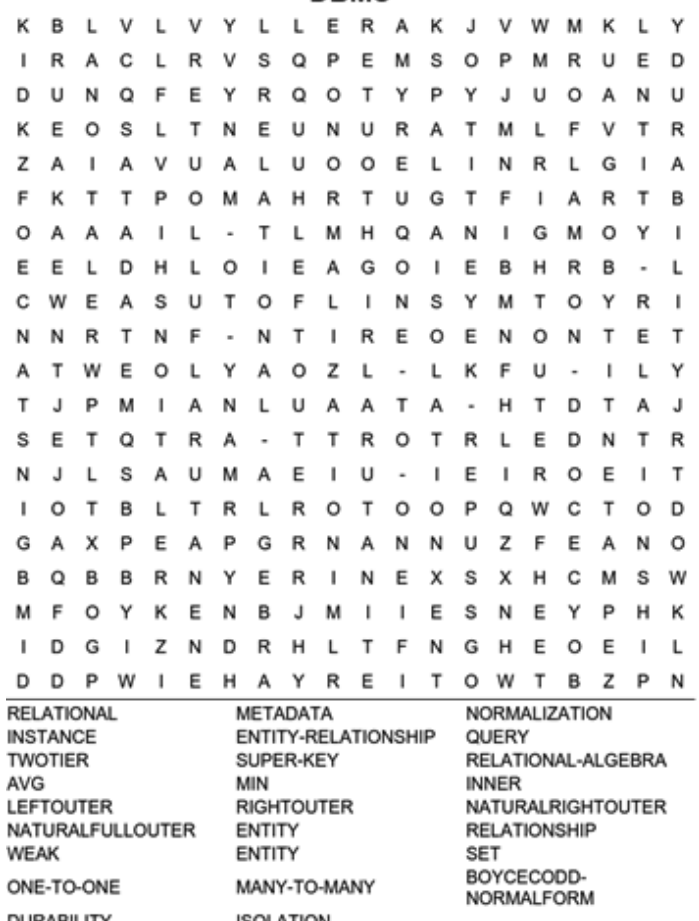

DURABILITY

\section{Fig. 3 : Findword}

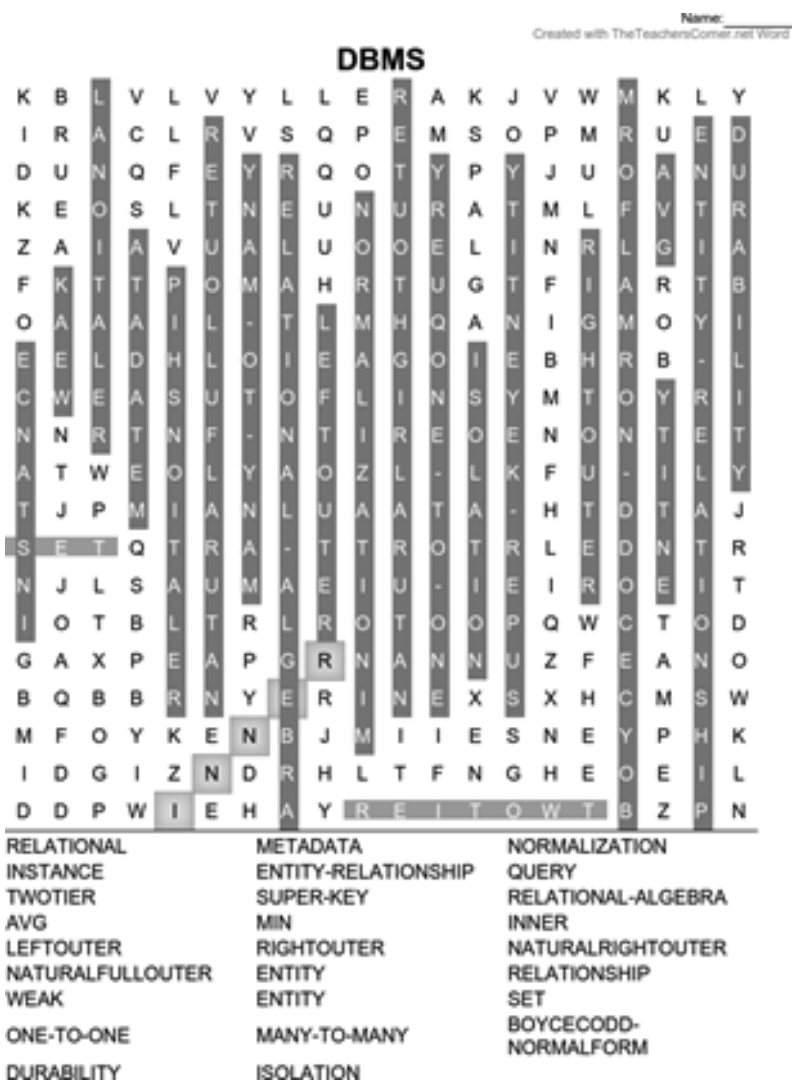

Fig. 4 : Findword with Answer

\section{Scramble Word}

The scramble word is scarcely homogeneous to findword with the only distinction is one have to find the solution within given word i.e. rearrange the given letters to make the technical word. Fig. 5 shows the example of scramble word and Fig. 6 shows the solution to it. One thing to be descried that the dependency can be set between crossword, findword and scramble word i.e. world got from crossword and scramble word to be probed in findword. This makes the student cerebrate beyond.

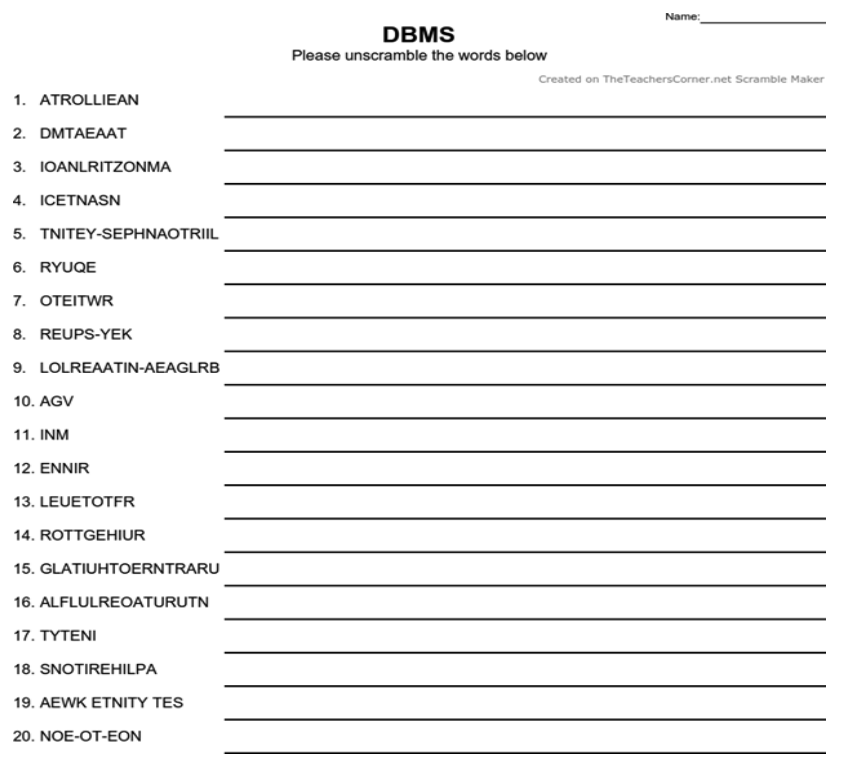

Fig. 5 : Scramble word

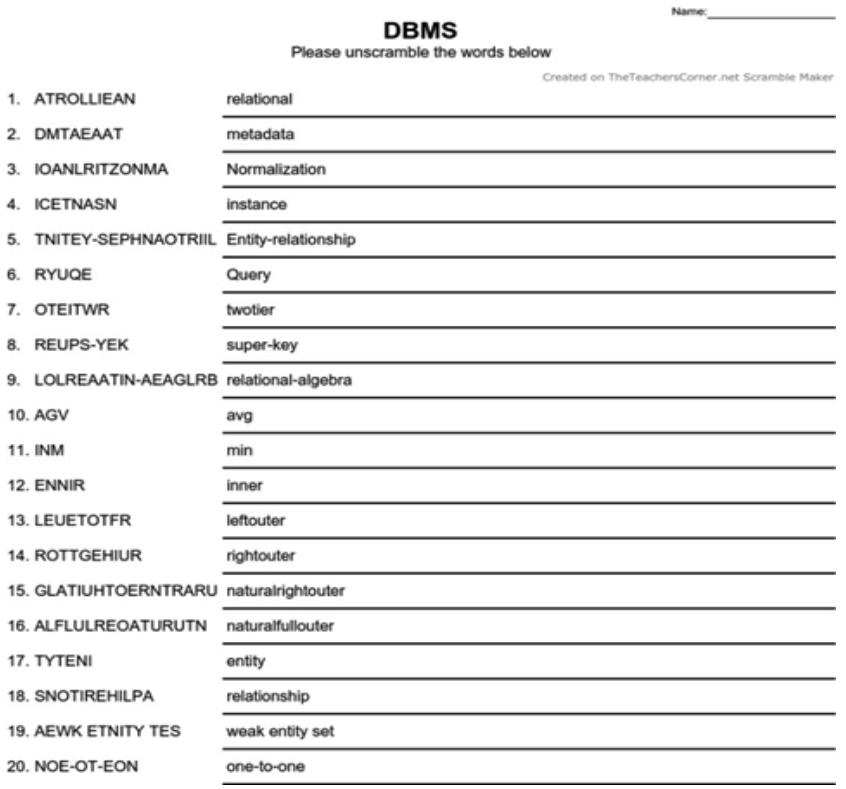

Fig. 6 : Scramble word with answer 


\section{Kahoot}

Kahoot (Kahoot, 2019) is a new game based technology that will be helping the students as today's generation is with mobile devices with high speed internet. One more advantage in Kahoot is that it can allow the already created (by someone else) to be utilized by others. At the end of the quiz it gives well structured reports for evaluators too. Fig. 7 shows the sample report of result summary.

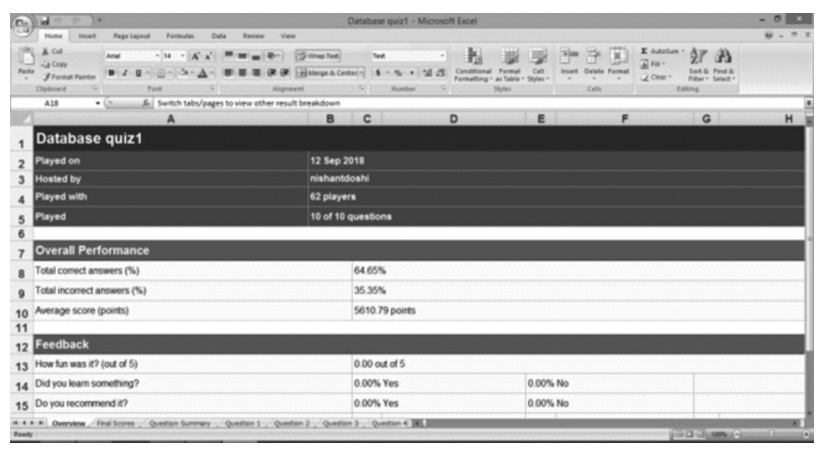

Fig. 7 : Kahoot Report

\section{Feedback}

After each of the technology, we have taken the feedback and based on rating we have done the average it over given assigned parameters as given in Table 1. Based on the analysis it shows that student finds it more useful to give test based on technology. The parameters are as follows. Each parameter is range over scale of $0-5$

I. Does this technology provide you learning environment

II. Does this technology made your subject concept clear

III. Does this technology helps you improvise communication skill

IV. Doesthis more useful in conveying the fundamental as to simple approach

V. Does this technology makes you boring as to simple approach

VI. Does this approach improvise your creative thinking as to the simple approach

VII. Does this approach time efficient in conveying the meaning as to simple approach
VIII. Do you want future tests based on this technology

IX. Do you want to utilize this technology in your future endeavours

X. Finally, have you feel relaxed during the test based on technology

Table 1 : Feedback result analysis

\begin{tabular}{|l|l|l|l|l|}
\hline & Crossword & Findword & Scrambleword & Kahoot \\
\hline I & 4.10 & 4.60 & 4.90 & 4.90 \\
\hline II & 4.30 & 4.30 & 4.20 & 4.90 \\
\hline III & 4.60 & 4.50 & 4.50 & 4.80 \\
\hline IV & 4.20 & 4.40 & 4.70 & 4.60 \\
\hline V & 4.40 & 4.90 & 4.30 & 4.70 \\
\hline VI & 4.90 & 4.60 & 4.60 & 4.80 \\
\hline VII & 4.30 & 4.20 & 4.90 & 4.80 \\
\hline VIII & 4.20 & 4.80 & 4.20 & 4.70 \\
\hline IX & 4.80 & 4.70 & 4.10 & 4.80 \\
\hline X & 4.90 & 4.80 & 4.00 & 4.70 \\
\hline
\end{tabular}

Based on the data from above table, we have done the following interpretation and observations in association with students. Being the online environment, Kahoot will be more favourite to students. However, it requires continuous active internet connection. Crossword, Findword, Scrambleword will also well known among students being a hard copy option.

\section{Research component in Education}

As per our study and practical analysis with today's student, it embarks that the conventional teaching method may not be effective to grasp for each student. On the other hand, various tools and techniques will make the student to think and understand topic easily. From the faculty perspective, it will be somewhat difficult while converting the subject topic in this type of medium. This ultimately lead to the research in education field which becomes necessary in upcoming years.

\section{Conclusions}

In this paper, we have given our experiments with teaching by technology and impart that the use of 
technology in teaching the key to success in the 21 st century. In the future, one can do a detailed survey as well as proposed the new key inventions in making the technology at next level. Finally based on effectiveness of this study and practical analysis, we came for conclusion to work all in this directions to make education as effective as possible and not as burden on the student.

\section{Acknowledgment}

The heading of the Acknowledgment section and the References section must not be numbered. The authors wish to thank A, B, C. This work was supported in part by a grant from XYZ.

\section{References}

[1] Borich, G. D. (1988). Effective teaching methods. Pearson Education India.

[2] Dianat, P. (2019, July). Interactive teaching methods of optoelectronics for enhancing engagement of under- represented groups. In Fifteenth Conference on Education and Training in Optics and Photonics: ETOP 2019 (Vol. 11143 , p. 111430K). International Society for Optics and Photonics.

[3] Tutu, O.A. (2019). Survey of ICT and Education in Africa: Cape Verde Country Report.

[4] Rao, O.R.S. (2013) Outcome based Engineering Education - Need of the Hour, The Journal of Engineering Education, 1-13.

[5] Welch, R., Hitt, J., Baldwin, J., Bentler, D., Clarke, D., \& Gross, S. (2001) The ExCEED Teaching Workshop: Hints to Successful Teaching, Proceedings of the 2001 American Society for Engineering Education Annual Conference \& Exposition.
[6] Prince, M.J., \& Felder, R.M. (2006) Inductive Teaching and Learning Methods: Definitions, Comparisons and Research Bases, Journal of Engineering Education, 123-138.

[7] Wankat P.C., Felder, R. M., Smith, K.A., \& Oreovicz, F.S. (2002) The Scholarship of Teaching and Learning in Engineering, Chapter 11 of M.T. Huber and S. Morreale, eds., Disciplinary Styles in the Scholarship of Teaching and Learning: Exploring Common Ground. AAHE/Carnegie Foundation for the Advancement of Teaching, Washington, 1-13.

[8] Kapranos, P., \& Tsakiropoulos, P. (2008) Teaching Engineering Students, International Symposium for Engineering Education, 2008, Dublin City University, Ireland.

[9] Houghton, W. (2004) Engineering Subject Centre Guide: Learning and Teaching Theory for Engineering Academics, The Higher Education Academy, Engineering Subject Centre, University of Exeter.

[10] Goodhew, P.J. (2010) Teaching Engineering All you need to know about engineering education but were afraid to ask, The Higher Education Academy - UK Centre for Materials Education, University of Liverpool, ISBN 9781-907207-22-8.

[11] Atasi Mohanty, Deepshikha Dash, "Engineering Education in India: Preparation of Professional Engineering Educators" , Journal of Human Resource and Sustainability Studies, 2016, 4, 92- 101

[12] Kahoot, https://kahoot.it accessed on 23-112018 